International Journal of Pure and Applied Mathematics

Volume 99 No. $4 \quad 2015,439-443$

ISSN: 1311-8080 (printed version); ISSN: 1314-3395 (on-line version)

url: http://www.ijpam.eu

doi: http://dx.doi.org/10.12732/ijpam.v99i4.4

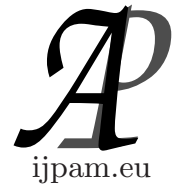

\title{
CHARACTERIZATIONS OF OPERATOR ORDER FOR THREE POSITIVE DEFINITE OPERATORS \\ VIA OPERATOR MEAN
}

\author{
Jian Shi $^{1}{ }^{\S}$, Siyuan Shen ${ }^{2}$ \\ ${ }^{1}$ College of Mathematics and Computer Science \\ Hebei University \\ Baoding, 071002, Hebei, P.R. CHINA \\ ${ }^{2}$ Department of Basic Sciences \\ Shijiazhuang Tiedao University Sifang College \\ Shijiazhuang, 051132, Hebei, P.R. CHINA
}

\begin{abstract}
Motivated by Lin and Cho's characterizations of $A \geq B \geq C$ via extended grand Furuta inequality, we present two characterizations of $A \geq B \geq$ $C$ via operator mean.
\end{abstract}

AMS Subject Classification: 47A63

Key Words: positive definite operator, Löwner-Heinz inequality, extend grand furuta inequality, operator order, operator mean

\section{Introduction}

A capital letter (such as $T$ ) stands for a bounded linear operator on a Hilbert space. $T>0$ and $T \geq 0$ mean $T$ is a positive definite operator and $T$ is a positive semidefinite operator, respectively.

As an important and historic extension of Löwner-Heinz inequality $(A \geq$ $\left.B \geq 0 \Rightarrow A^{\alpha} \geq B^{\alpha}, \alpha \in[0,1]\right), \mathrm{T}$. Furuta proved the following theorem in 1987.

Received: August 20, 2014

(C) 2015 Academic Publications, Ltd.

$\S$ Correspondence author

url: www.acadpubl.eu 
Theorem 1.1. (see Furuta Inequality, [2, 7]) If $A \geq B \geq 0$, then $\left(A^{\frac{r}{2}} A^{p} A^{\frac{r}{2}}\right)^{\frac{1}{q}} \geq$ $\left(A^{\frac{r}{2}} B^{p} A^{\frac{r}{2}}\right)^{\frac{1}{q}}$ holds for $p \geq 0, r \geq 0, q \geq 1$ with $(1+r) q \geq p+r$.

In 1995, T. Furuta obtained the following grand form of Theorem 1.1.

Thoerem 1.2. (see Grand Furuta Inequality, $[3,8]$ ) If $A \geq B \geq 0$ with $A>0$, then $A^{1-t+r} \geq\left[A^{\frac{r}{2}}\left(A^{-\frac{t}{2}} B^{p} A^{-\frac{t}{2}}\right)^{s} A^{\frac{r}{2}}\right]^{\frac{1-t+r}{(p-t) s+r}}$ holds for $p, s \geq 1, t \in[0,1]$ and $r \geq t$.

In 2003, M. Uchiyama showed the following extended form of Theorem 1.2.

Theorem 1.3. (Extended Grand Furuta Inequality, [9]) If $A \geq B \geq C \geq 0$ with $B>0$, then $A^{1-t+r} \geq\left[A^{\frac{r}{2}}\left(B^{-\frac{t}{2}} C^{p} B^{-\frac{t}{2}}\right)^{s} A^{\frac{r}{2}}\right]^{\frac{1-t+r}{(p-t) s+r}}$ holds for $p, s \geq 1$, $t \in[0,1]$ and $r \geq t$.

For $S, T>0$, operator mean of $S$ and $T$ is defined by F. Kubo and T. Ando in [4] as $S \sharp_{\alpha} T=S^{\frac{1}{2}}\left(S^{-\frac{1}{2}} T S^{-\frac{1}{2}}\right)^{\alpha} S^{\frac{1}{2}}$, where $\alpha \in[0,1]$. Generally, if $\alpha \in \mathbb{R}$, $S^{\frac{1}{2}}\left(S^{-\frac{1}{2}} T S^{-\frac{1}{2}}\right)^{\alpha} S^{\frac{1}{2}}$ is denoted by $S \bigsqcup_{\alpha} T$.

Recently, C. -S. Lin and Y. J. Cho in [5] showed characterizations of $A \geq$ $B \geq C$ via extended grand Furuta inequality. Motived by [5], we present two characterizations of $A \geq B \geq C$ via operator mean.

\section{Main Results}

C.-S. Lin in 2010 showed the following results on operator mean.

Lemma 2.1. (see [6]) For $p, s \geq 1, t \in[0,1]$ and $r \geq 1$, if $A \geq B \geq C>0$, then

$$
C^{r} \sharp \frac{r-1}{(p-t) s+r}\left(B^{\frac{t}{2}} A^{-p} B^{\frac{t}{2}}\right)^{s} \leq C \leq B \leq A \leq A^{r} \sharp \frac{r-1}{(p-t) s+r}\left(B^{\frac{t}{2}} C^{-p} B^{\frac{t}{2}}\right)^{s} .
$$

Lemma 2.2. (see [6]) For $p, s \geq 1, t \in[0,1]$ and $r \geq 1+t$, if $A \geq B \geq C>$ 0 , then

$$
\begin{aligned}
& C^{r-t_{\sharp} \frac{r-1-t}{(p-t) s+r}} C^{-\frac{t}{2}} B^{\frac{t}{2}}\left(B^{-t} \bigsqcup_{s} A^{-p}\right) B^{\frac{t}{2}} C^{-\frac{t}{2}} \\
\leq & C \leq B \leq A \\
\leq & A^{r-t_{\sharp}}
\end{aligned}
$$


Next we will show two characterizations of operator order for three positive definite operators via Lemma 2.1 and Lemma 2.2.

Theorem 2.1. For $A, B, C>0$. $A \geq B \geq C$ if and only if the following two inequalities

$$
\begin{gathered}
C^{r} \sharp \frac{r-1}{(p-t) s+r}\left(B^{\frac{t}{2}} A^{-p} B^{\frac{t}{2}}\right)^{s} \leq C, \\
A \leq A^{r} \sharp \frac{r-1}{(p-t) s+r}\left(B^{\frac{t}{2}} C^{-p} B^{\frac{t}{2}}\right)^{s}
\end{gathered}
$$

hold for $p, s \geq 1, t \in[0,1]$ and $r \geq 1$.

Theorem 2.2. For $A, B, C>0$. $A \geq B \geq C$ if and only if the following two inequalities

$$
\begin{aligned}
& C^{r-t_{\sharp} \frac{r-1-t}{(p-t) s+r}} C^{-\frac{t}{2}} B^{\frac{t}{2}}\left(B^{-t} \natural_{s} A^{-p}\right) B^{\frac{t}{2}} C^{-\frac{t}{2}} \leq C, \\
& A \leq A^{r-t_{\sharp} \frac{r-1-t}{(p-t) s+r}} A^{-\frac{t}{2}} B^{\frac{t}{2}}\left(B^{-t} \natural_{s} C^{-p}\right) B^{\frac{t}{2}} A^{-\frac{t}{2}}
\end{aligned}
$$

hold for $p, s \geq 1, t \in[0,1]$ and $r \geq 1+t$.

Proof of Theorem 2.1. The necessity is obviously by Lemma 2.1. We only need to prove the sufficiency. We adopt the same method as in [5].

Putting $p=t=1, r=2$ in $(2.1)$, we have $C^{2} \sharp_{\frac{1}{2}}\left(B^{\frac{1}{2}} A^{-1} B^{\frac{1}{2}}\right)^{s} \leq C$. By the definition of $\sharp$, the following inequality holds.

$$
\left(C^{-1}\left(B^{\frac{1}{2}} A^{-1} B^{\frac{1}{2}}\right)^{s} C^{-1}\right)^{\frac{1}{2}} \leq C^{-1} .
$$

Because $C>0$ and $C$ is bounded, there exist two positive numbers $m_{C}$ and $n_{C}$ such that $m_{C} I \geq C \geq n_{C} I>0$. According to Theorem 6 in [1] $(X \geq Y \geq 0$ with $\left.m I \geq X \geq n I>0 \Rightarrow \frac{(m+n)^{2}}{4 m n} X^{2} \geq Y^{2}\right)$, we have

$$
C^{-1}\left(B^{\frac{1}{2}} A^{-1} B^{\frac{1}{2}}\right)^{s} C^{-1} \leq \frac{\left(m_{c}^{-1}+n_{c}^{-1}\right)^{2}}{4 m_{c}^{-1} n_{c}^{-1}} C^{-2} .
$$

Deleting $C^{-1}$ in the both side of the inequality above, and applying LöwnerHeinz inequality, the following inequality holds.

$$
B^{\frac{1}{2}} A^{-1} B^{\frac{1}{2}} \leq\left(\frac{\left(m_{c}^{-1}+n_{c}^{-1}\right)^{2}}{4 m_{c}^{-1} n_{c}^{-1}}\right)^{\frac{1}{s}} I .
$$

Letting $s \rightarrow+\infty$ above, then $A^{-1} \leq B^{-1}$, which ensures $A \geq B$. 
By the same way, we can obtain $B \geq C$ from (2.2).

Proof of Theorem 2.2. The necessity is obviously by Lemma 2.2. We only need to prove the sufficiency.

Putting $p=t=1, r=4$ in $(2.3)$, we have $C^{3{ }_{H_{1}}} C^{-\frac{1}{2}} B^{\frac{1}{2}}\left(B^{-1} \natural_{s} A^{-1}\right) B^{\frac{1}{2}} C^{-\frac{1}{2}} \leq$ $C$. By the definitions of $\sharp$ and $\downarrow$, the following inequality holds.

$$
\left(C^{-2}\left(B^{\frac{1}{2}} A^{-1} B^{\frac{1}{2}}\right)^{s} C^{-2}\right)^{\frac{1}{2}} \leq C^{-2} .
$$

According to Theorem 6 in [1], we have

$$
C^{-2}\left(B^{\frac{1}{2}} A^{-1} B^{\frac{1}{2}}\right)^{s} C^{-2} \leq\left(\frac{\left(m_{c}^{-2}+n_{c}^{-2}\right)^{2}}{4 m_{c}^{-2} n_{c}^{-2}}\right)^{\frac{1}{s}} C^{-4} .
$$

Deleting $C^{-2}$ in the both side of the inequality above, and applying LöwnerHeinz inequality, the following inequality holds.

$$
B^{\frac{1}{2}} A^{-1} B^{\frac{1}{2}} \leq\left(\frac{\left(m_{c}^{-2}+n_{c}^{-2}\right)^{2}}{4 m_{c}^{-2} n_{c}^{-2}}\right)^{\frac{1}{s}} I .
$$

Letting $s \rightarrow+\infty$ above, then $A^{-1} \leq B^{-1}$, which ensures $A \geq B$.

By the same way, we can obtain $B \geq C$ from (2.4).

Remark 2.1. We can also obtain $A \geq B$ from (2.5) and (2.8) by Theorem 3.1 in [10](For $C, D>0, r>0, \delta>-r$ and $0<w \leq 1$, if $C^{\delta+r} \geq\left(C^{\frac{r}{2}} D^{s} C^{\frac{r}{2}}\right)^{w}$ holds for any $s>1$, then $D \leq I$ ). We leave the details to readers.

\section{References}

[1] M. Fujii, S. Izumino, R. Nakamoto, Y. Seo, Operator inequalities related to Cauchy-Schwarz and Hölder-McCarthy inequalities, Nihonkai Math. J., 8 (1997), 117-122.

[2] T. Furuta, $A \geqslant B \geqslant O$ assures $\left(B^{r} A^{p} B^{r}\right)^{1 / q} \geqslant B^{(p+2 r) / q}$ for $r \geqslant 0, p \geqslant 0$, $q \geqslant 1$ with $(1+2 r) q \geqslant p+2 r$, Proc. Amer. Math. Soc., 101 (1987), 85-88.

[3] T. Furuta, Extension of the Furuta inequality and Ando-Hiai log majorization, Linear Algebra Appl., 219 (1995), 139-155.

[4] F. Kubo, T. Ando, Means of positive linear operators, Math. Ann., 246 (1980), 205-224. 
[5] C.-S. Lin, Y.J. Cho, Characterizations of operator inequality $A \geq B \geq C$, Math. Inequal. Appl., 14 (2011), 575-580.

[6] C.-S. Lin, On operator inequalities in terms of geometric mean, International J. Puar Appl. Math., 58 (2010), 299-308.

[7] K. Tanahashi, Best possibility of the Furuta inequality, Proc. Amer. Math. Soc., 124 (1996), 141-146.

[8] K. Tanahashi, The best possibility of the grand Furuta inequality, Proc. Amer. Math. Soc., 128 (2000), 511-519.

[9] M. Uchiyama, Criteria for operator mean, J. Math. Soc. Japan., 55 (2003), 197-207.

[10] J. Yuan, C. Wang, Riccati type operator equation and Furuta's question, Math. Inequal. Appl., Prerpint. 
